\title{
¿Puede un programa de ocho semanas basado en la técnica hipopresivaproducir cambios en la función del suelo pélvico y composición corporal de jugadoras de rugby? Can an eight-week program based on the hypopressive technique produce changes in pelvic floor function and body composition in female rugby players?
}

\author{
Myrian Marta Álvarez Sáez*, Tamara Rial Rebullido**, Iván Chulvi Medrano***, Jose Luis García Soidán****, Juan Manuel
} Cortell Tormo*****

*Clinica Maio Fisioterapia (España), **International Hypopressive \& Physical Therapy Institute (España), ***Centro Benestar (España),

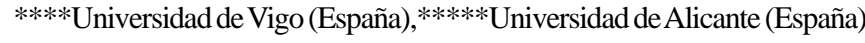

\begin{abstract}
Resumen. La Técnica Hipopresiva (TH) se basa en posturas de estiramiento sostenidas mientras se realizan respiraciones torácicas y apneas intermitentes. Existe poca evidencia sobre sus efectos en la musculatura abdominal y del Suelo Pélvico (SP), así como en otras variables cineantropométricas, especialmente en mujeres deportistas. El objetivo del estudio fue describir los efectos de un programa basado en la TH sobre la composición corporal, contracción del transverso del abdomen (TA) y función muscular del SP de mujeres deportistas. La muestra estuvo formada por 11 jugadoras de rugby (23.3años, $\mathrm{SD}=2,69$; 23.3; IMC, $\mathrm{SD}=1,98$ ) que realizaron ocho posturas de la $\mathrm{TH}$, realizadas en sesiones de 45 minutos, dos veces por semana a lo largo de ocho semanas. Todas las sesiones fueron supervisadas por una fisioterapeuta cualificada. Se evaluó la composición corporal a través de impedancia bioeléctrica y la circunferencia de la cadera y cintura se midieron con cinta antropométrica. La máxima contracción del TA se evalúo con biofeedback de presión. Para analizar la tonicidad y contractilidad muscular del SP se utilizó un pelvímetro Phenix. Se observaron diferencias significativas tras el periodo de entrenamiento en la circunferencia de la cintura, la máxima contracción del TA y en la contractilidad y tonicidad de la musculatura del SP $(p<.05)$. No se encontraron diferencias significativas en los valores de composición corporal y en la circunferencia de la cadera $(p>$.05). Estos resultados preliminares indican que un programa supervisado de ocho semanas de la TH puede ser utilizado para mejorar la función abdominal y del SP de jugadoras de rugby.
\end{abstract}

Palabras clave. ejercicio terapéutico, mujer, deporte, incontinencia urinaria de esfuerzo, músculos abdominales

Abstract. The hypopressive technique (HT) uses active muscle stretching postures in conjunction with thoracic breathing and intermittent breath holding. There is little evidence about the effects of HT on the deep musculature of the abdomen and the pelvic floor (PF) as well as other kinanthropometric variables, especially in female athletes. The aim of this study was to examine the effects of HT program on body composition, contraction of the transversus abdominis (TA) and PF muscle function in female athletes. 11 female rugby players (23.3yr, SD=2.69; 23.3; BMI, $\mathrm{SD}=1.98$ ) performed eight HT postures twice per week for 8 weeks with a total duration of 45 -minutes per session. All training sessions were supervised by a qualified HT physiotherapist. Body composition was evaluated through bioelectrical impedance and waist and hip circumferences were measured with anthropometric tape. Maximum contraction of the TA was assessed with a biofeedback pressure device. The pelvimetre Phenix was used to analyze tonicity and PF contractility. Following the training period, significant differences were observed in waist circumference, maximum contraction of the TA and contractility and muscle tone of the PF $(p<.05)$. No significant differences were found in body composition and hip circumference $(p>.05)$. These preliminary findings indicate that a supervised 8-week program of HT can be used to enhance abdominal and perineal function in female rugby players.

Key words. exercise therapy, women, sport, stress urinary incontinence, abdominal muscles.

\section{Introducción}

La Técnica Hipopresiva (TH) se compone de ejercicios respiratorios y de estiramiento postural (Rial \& Pinsach, 2014), que se ejecutan durante un cierto tiempo en diferentes posturas mantenidas en isometría y/o contracción excéntrica. Simultáneamente, se realizan respiraciones torácicas profundas intercaladas con apneas espiratorias, asociadas a apertura de la caja torácica. Durante su ejecución se ha registrado activación de la musculatura del Suelo Pélvico (SP) y del Transverso Abdominal (TA) (Stüpp, Resende, Petricelli, Nakamura, Alexandre \& Zanetti, 2011).

Esta TH fue inicialmente utilizada en el norte de Europa, como ejercicio de rehabilitación abdominal en el postparto. Se aplicaba en el tratamiento de disfunciones del SP como el prolapso de órganos pélvicos (Bernardes et al., 2012). En este sentido, se aconsejó su uso como recurso específico para mujeres con poca propiocepción de la musculatura del SP (Latorre, Seleme, Resende, Stüpp \& Berghmans, 2011).

Si bien, en los últimos años, ha sido popularizada en España como práctica de actividad físico-saludable con una presencia cada vez mayor en los centros deportivos y de fitness (Rial \& Pinsach, 2014). Sin embargo, no existen evidencias de sus posibles efectos en la condición física saludable de diferentes poblaciones. De momento, las referencias encontradas en la literatura, están orientadas o bien a la rehabilitación de patologías

Fecha recepción: 17-07-15. Fecha de aceptación: 07-12-15

Tamara Rial Rebullido

rialtamara@gmail.com perineales (Resende et al., 2011; Rial, Chulvi-Medrano, CortellTormo \& Álvarez, 2015) o bien a patologías de columna vertebral (Caufriez, Fernández \& Brynhildsvoll, 2011). Por ello, su uso se podría extrapolar a un contexto de prevención de disfunciones del SP para la mujer deportista.

La función del SP durante el ejercicio o el deporte aún no está bien definida. Por un lado se hipotetiza que el ejercicio de impacto puede debilitar el SP como resultado de repetitivos incrementos de presión intra-abdominal (Bo, 2004) y por el otro alterar su función (Da Roza et al., 2015; Kruger, Dietz \& Murphy, 2007) por cambios morfológicos (Kruger, Murphy \& Heap, 2005) o biomecánicos (Dietz \& Shek, 2008). En este sentido, la literatura muestra una alta incidencia de incontinencia urinaria de esfuerzo en mujeres que practican deportes de alta intensidad e impacto (Goldstick \& Constantini, 2014). La pérdida de orina durante la práctica deportiva es una condición que puede limitar la participación e incluso el rendimiento de la deportista (Brown \& Miller, 2001). La frecuencia o intensidad en el entrenamiento han sido identificados como factores que incrementan la prevalencia de incontinencia urinaria de esfuerzo (Eliasson, Edner, \& Mattsson, 2008). El rugby es un ejemplo de deporte de alta intensidad que puede derivar en un factor de riesgo de incontinencia urinaria de esfuerzo debido a las frecuentes situaciones de impacto (Nygaard, 1997)

El rugby se caracteriza por ser un deporte intermitente de colisión practicado mundialmente por ambos sexos (Jones, Emmonds, Hind, Nicholson, Rutherford \& Till, 2015). El índice de lesión deportiva más frecuente es de cabeza, cuello y espalda, así como hombro y miembros inferiores (Gabbett, 2003; King, Hume, Milburn \& Guttenbeil, 2010). Este tipo de lesio- 
nes principalmente de origen traumático, acontecen entre deportistas independientemente de su edad o la categoría de la competición (Gabett, 2008). Por ello, resulta necesario identificar los factores de riesgo lesional para poder implementar y evaluar un programa de prevención efectivo (Gabbett, King \& Jenkins, 2008). Así mismo, se deben tener en cuenta factores relacionados con el desarrollo de las aptitudes físicas como es la composición corporal del deportista. Estudios realizados con jugadoras de rugby elite revelaron una correlación negativa entre el porcentaje de grasa total y su rendimiento deportivo (Gabett, 2007; Jones et al., 2015). Estos resultados sugieren que una reducción del porcentaje de masa grasa como incremento de la masa muscular total podrían incrementar su rendimiento (Jones et al., 2015).

De esta forma y teniendo en cuenta lo anteriormente expuesto, la principal hipótesis de nuestro trabajo fue que el entrenamiento con la TH podría constituir una alternativa de ejercicio tanto para mejorar la función muscular del SP como la composición corporal en el rugby femenino. Puesto que hasta la actualidad no existen estudios sobre estas variables, el objetivo planteado fue comprobar los efectos de un programa de la TH sobre parámetros de composición corporal y de función muscular del SP, en un grupo de mujeres deportistas que practican rugby.

\section{Metodología}

\section{Diseño}

Se ha diseñado un estudio cuasi-experimental, con diseño transversal, en un grupo piloto, con medidas pre y post test para un solo grupo de intervención. La recogida de datos tuvo lugar entre los meses de Marzo y Mayo en la Clínica Maio de Vigo (España). El marco muestral estuvo compuesto por jugadoras convocadas por la Selección Gallega de Rugby para el Campeonato Nacional de Selecciones Territoriales. Tras haber firmado el consentimiento informado sobre los riesgos y beneficios del estudio, tomaron parte en el estudio 11 jugadoras convocadas por la selección, siendo excluidas 3 jugadoras por no haber cumplido los criterios de inclusión. Los criterios de inclusión fueron: i) nulíparas ii) no tener contraindicaciones relativas y/o absolutas para la práctica de actividad física y/o deporte; iii) no estar embarazada; iv) no ser hipertensa. Los criterios de exclusión fueron incapacidad física para realizar la $\mathrm{TH}$, no haber mantenido relaciones sexuales plenas y no cumplir algunos de los criterios de inclusión anteriormente descritos. Las características de la muestra pueden observarse en la

Tabla 1.

\begin{tabular}{cc}
\hline Características de las participantes. & \\
\hline Variable & Media (DE) \\
$\mathrm{n}=11$ \\
\hline Edad (años) & $23.36(2.69)$ \\
IMC (kg/m2) & $23.33(1.98)$ \\
\hline
\end{tabular}

tabla 1.

\section{Protocolo}

Todas las participantes leyeron y firmaron el consentimiento de participación en la investigación, cuyo procedimiento se realizó respetando los principios éticos de la declaración de Helsinki (Rev. 2008) y la Ley de Protección de Datos 15/1999, el proyecto de investigación fue aprobado por el Comité ético local.

Durante la primera visita a la clínica las jugadoras firmaron el consentimiento informado y tuvieron una sesión de familiarización con los ejercicios incluidos en el programa de TH. En la segunda visita fueron realizadas las valoraciones de composición corporal, teniendo en cuenta todas las recomendaciones para la estandarización de las mismas. En una tercera visita se llevaron a cabo los tests de competencia muscular. A las 48 horas de la última valoración se aplicó a la muestra una intervención de 8 semanas de TH con una frecuencia de 2 sesiones semanales. Dentro de las 24 horas siguientes a finalizar el período experimental, se repitió toda la batería de evaluaciones iniciales.

Se recogieron los datos de las siguientes variables del estudio: composición corporal (cambios sobre el porcentaje de grasa y masa muscular); perímetro de cintura y cadera; competencia del transverso del abdomen; función muscular del SP. Con cada participante se siguió estrictamente la siguiente secuencia de valoraciones:

\section{a. Medidas antropométricas.}

La altura fue calculada con un tallímetro (modelo SECA) con un rango de medida 80 a 200 cm y precisión de 1 milímetro. Se siguieron los procedimientos cineantropométricos recomendados por (Esparza, 1993), donde se indica que la medición requiere que la participante se encuentre en bipedestación permaneciando con los talones, glúteos y espalda en contacto con el plano vertical del tallímetro y la cabeza en el plano de Frankfurt. El registro fue expresado en centímetros. La medición de la circunferencia de cintura y cadera se llevó a cabo utilizando una cinta antropométrica (modelo Harpenden, precisión $\pm 1 \mathrm{~mm}$ ). El protocolo de medición se basó en el descrito por Esparza. El perímetro de cintura se midió utilizando como referencia el punto medio entre el borde costal y la cresta ilíaca y el perímetro de cadera a la altura del trocánter mayor del fémur. Las mediciones se expresaron en centímetros.

La composición corporal se estudió mediante impedancia bioeléctrica que mide la resistencia al flujo de distintas corrientes eléctricas por las regiones corporales (García-Soidán, López, Ogando, Fernández, Padrón \& Prieto, 2014). Ha sido evaluada mediante un impedanciómetro con sistema de medición octopolar con un espectro de medición $50 \mathrm{Khz}$ (Fabricante Tanita modelo BC-418MA $\left.{ }^{\circledR}\right)$. Este instrumento dispone de ocho electrodos para realizar el análisis de la composición corporal de todo el cuerpo y por segmentos. La toma de datos se realizó siguiendo los protocolos estandarizados por Heyward \& Wagner (2004).

\section{b. Competencias musculares.}

Se utilizó un biofeedback de presión (Stabilizer Chattanooga Group, $\left.{ }^{\circledR}\right)$ para evaluar indirectamente la habilidad de contracción del TA, mediante una maniobra de hundimiento abdominal a través del test de aislamiento del TA (Hodges et al., 1996). Esta herramienta ha sido comprobada como válida y fiable para evaluar la función de la musculatura profunda del abdomen (Cairns, Harrison, \& Wright, 2000). Para efectuar el test se solicitó a la participante que se colocara en decúbito prono sobre la camilla, brazos estirados a lo largo del cuerpo y la cabeza a un lado. Se situó el biofeedback inflado a $20 \mathrm{mmHg}$ debajo del abdomen, centrado entre parrilla costal y las espinas ilíacas anterosuperiores. Tras el registro en situación de reposo, se solicitaba un hundimiento abdominal, con ausencia de basculación de pelvis o contracción de glúteos.

La medición de la contractilidad del SP fue valorada mediante un Pelvímetro Phenix, (VITALIS Electronic Concept Lignon Innovation ${ }^{\circledR}$, en 93/42 89/336/CEE). La evaluación se llevó a cabo con la participante en posición ginecológica, con la cadera y piernas flexionadas a $90^{\circ}$ en decúbito supino. Las participantes fueron instruidas previamente para contraer su musculatura perineal con la percepción máxima de esfuerzo y a mantener la contracción durante 10 segundos y en ausencia de la contracción simultánea de otra musculatura.

A continuación, se midió la tonicidad y contractilidad muscular del SP respectivamente. Durante la valoración tonimétrica las ramas del pelvímetro someten al SP a un estiramiento pasivo hasta $15^{\circ}$ de apertura. Para la valoración contráctil la examinadora abre y fija el instrumento a $5^{\circ}$ de abertura. Seguidamente, 
se pide una contracción del SP durante 10 segundos contra una resistencia máxima impuesta por el bloqueo de las ramas del pelvímetro (contracción isométrica). Tras esta contracción se permite un descanso de unos 20 segundos y posteriormente se pide una segunda contracción máxima. Es realizada una media de los dos valores expresando la medida en gramos por centímetros cuadrados $\left(\mathrm{gr} / \mathrm{cm}^{2}\right)$.

\section{Programa de ejercicio físico}

El protocolo de la TH fue aplicado durante 8 semanas a razón de 2 días semanales. La extensión de cada sesión realizando todos los ejercicios incluidos fue de 45 minutos. Todas las sesiones fueron dirigidas y supervisadas por una fisioterapeuta especializada en SP y certificada en esta metodología. La secuencia de ejercicios mantuvo las indicaciones técnicas y posturales: i) Crecimiento axial de la columna; ii) Rectificación cervical; iii) Adelantamiento del eje corporal; iv) Decoaptación de la cintura escapular; v) Ligera flexión de rodillas; vi) Ligera flexion dorsal de tobillos.

Se realizó un protocolo básico de ocho posturas que pueden apreciarse en la figura 1. En cada posición se realizaban tres respiraciones torácicas lentas seguidas de una apnea espiratoria acompañada de máxima apertura costal. La apnea se mantenía un máximo de 25 segundos. Cada ejercicio fue repetido tres

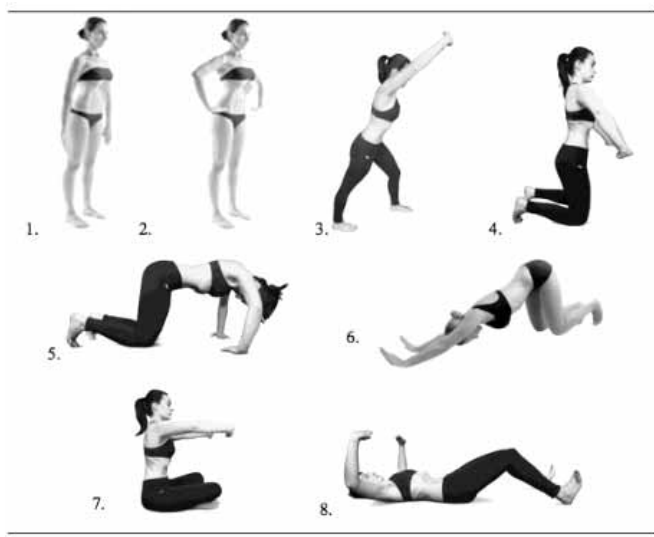

Figura 1. Posiciones de los ejercicios hipopresivos del protocolo. Elaboración Propia. veces.

\section{Análisis Estadístico}

Todo el análisis de datos se realizó con el paquete estadístico SPSS para Windows (versión 17.0). Los datos descriptivos se presentan como números o medias con desviación estandar (DE). La normalidad de cada una de las variables analizadas se realizó mediante la prueba de Shapiro-Wilk. Para analizar las diferencias entre los dos momentos de evaluación fue utilizado el test Wilcoxon. El nivel de significancia estadística fue establecido en $p<.05$.

\section{Resultados}

La tabla 2 muestra los resultados tras la realización del programa de ocho semanas de la TH entre los dos momentos de evaluación, en donde se observan diferencias significativas para el perímetro de cintura $(Z=2.68 ; p=.007)$ y la eficacia en la contracción del TA ( $Z=2.81 ; p=.005)$. Los valores de medición del SP, mostraron cambios significativos, tanto para la contractilidad como tonicidad muscular $(Z=2,36 ; p=.018)$. En la tabla 3 se muestran los datos de las variables de composición corporal en los cuales no se mostraron cambios significativos ( $p>.05)$.

\section{Discusión}

La discusión de los resultados resulta compleja pues, para
Tabla

Mediciones antes y después del programa para las variables antropométricas y competencias musculares.

\begin{tabular}{|c|c|c|c|c|}
\hline \multirow{2}{*}{ Variable } & \multirow{2}{*}{$\begin{array}{c}\text { Pretest } \\
\text { M (DE) }\end{array}$} & \multirow{2}{*}{$\begin{array}{l}\text { Postest } \\
\text { M (DE) }\end{array}$} & \multicolumn{2}{|c|}{ Efecto entre momentos } \\
\hline & & & $\mathrm{Z}$ & $P$ \\
\hline Perímetro de cadera $(\mathrm{cm})$ & $97.56(5.15)$ & $97.11(4.93)$ & .76 & .446 \\
\hline Perímetro de cintura $(\mathrm{cm})$ & $77.33(5.26)$ & $74.44(5.57)$ & 2.68 & $* .007$ \\
\hline Contracción TA (mmHg) & $63.64(16.89)$ & 49.09 (10.55) & 2.81 & $* .005$ \\
\hline Contracción SP (gr/ $\left./ \mathrm{cm}^{2}\right)$ & $1165.29(510.85)$ & 1453.14 (529.69) & 2.36 & $* .018$ \\
\hline Tonicidad SP $\left(\mathrm{gr} / \mathrm{cm}^{2}\right)$ & 212.57 (114.74) & $329.00(92.82)$ & 2.36 & $* .018$ \\
\hline \multicolumn{5}{|c|}{$\begin{array}{l}\text { Tabla } 3 . \\
\text { Mediciones antes y después del programa para las variables de Impedancia Bioeléctrica. }\end{array}$} \\
\hline & Pretest & Postest & Efecto entr & nentos \\
\hline Variable & $\mathrm{M}(\mathrm{DE})$ & $\mathrm{M}(\mathrm{DE})$ & $\mathrm{Z}$ & $p$ \\
\hline IMC $(\mathrm{kg} / \mathrm{m} 2)$ & $23.36(2.11)$ & $23.33(2.34)$ & .447 & .655 \\
\hline MG total (\%) & $23.36(2.11)$ & $27.33(5.54)$ & .776 & .438 \\
\hline MG total (Kg) & $19.09(6.41)$ & $18.33(5.22)$ & 1.703 & .089 \\
\hline MM (\%) & $46.86(3.40)$ & $47.61(3.82)$ & 1.12 & .260 \\
\hline MG Tronco (\%) & $\begin{array}{l}24.36 \\
(5.29)\end{array}$ & $\begin{array}{l}24.11 \\
(6.73)\end{array}$ & .933 & .351 \\
\hline MG MSD (\%) & $\begin{array}{l}28.00 \\
(5.27)\end{array}$ & $\begin{array}{l}27.56 \\
(6.56)\end{array}$ & .763 & .445 \\
\hline MG MSI (\%) & $\begin{array}{l}28.45 \\
(5.20)\end{array}$ & $\begin{array}{l}28.11 \\
(6.48)\end{array}$ & .509 & .611 \\
\hline MG MID (\%) & $\begin{array}{l}32.18 \\
(2.82)\end{array}$ & $\begin{array}{l}31.67 \\
(3.53)\end{array}$ & .923 & .356 \\
\hline MG MII (\%) & $\begin{array}{l}32.55 \\
(2.87)\end{array}$ & $\begin{array}{c}32.11 \\
(3.51)\end{array}$ & .595 & .552 \\
\hline Agua total (\%) & $\begin{array}{l}34.36 \\
(2.50)\end{array}$ & $\begin{array}{l}34.37 \\
(2.72)\end{array}$ & .541 & .589 \\
\hline
\end{tabular}

Derecho; MSI= Miembro Superior Izquierdo; MID=Miembro Inferior Derecho; MII= Miembro Inferior Izquierdo

nuestro mejor conocimiento, es el primer estudio conocido con mujeres deportistas de élite que se adhiere a un programa regular de la TH.

En primer lugar, uno de los hallazgos más destacables fue que el perímetro de cintura abdominal disminuyó sin mostrarse cambios significativos en la masa grasa segmental del tronco. Estos datos pueden relacionarse con una mejora en la eficacia de activación de la musculatura profunda del abdomen durante la realización de la TH (Stupp et al., 2011).

La mejora obtenida en los patrones de contracción del TA, resulta indicativo de la mejora en una porción de la estabilidad lumbar del core, el cual es considerado un factor de riesgo modificable de gran importancia, tanto para las lesiones de la propia región lumbar como de los miembros inferiores (Wilkerson, Giles \& Seibel, 2012; Zazulak, Cholewicki \& Reeves, 2008). En una línea similar, se han descrito mejoras tras realizar un programa de diez semanas de ejercicio para la estabilidad lumbopélvica evaluado mediante un biofeedback de presión, en un grupo de jugadoras de baloncesto y de voleibol (Mills, J., Taunton \& Mills, W., 2005).

Tanto la eficacia en la contracción del SP como su tonicidad mejoraron tras la intervención. La terapia con programas específicos de SP ha mostrado ser efectiva en jóvenes nulíparas deportistas (Da Roza, et al., 2012). Un estudio aleatorizado controlado (Resende et al., 2011) en mujeres con disfunción de SP con prolapso de órganos pélvicos, encontró que tanto el grupo de TH, como el grupo de ejercicios de contracción del SP más la TH, mejoraron la resistencia y la máxima contracción voluntaria del SP, aunque añadir la TH a los ejercicios de contracción de SP, no añadió mejoras en su función muscular.

Respecto a la composición corporal, no se obtuvieron cambios significativos tanto para el porcentaje de grasa corporal ni para el de masa muscular total. La TH consiste en un conjunto sistemático de ejercicios posturales y respiratorios asociados a un ritmo de trabajo respiratorio concreto (Rial \& Pinsach, 2014). $\mathrm{Al}$ ser un programa de ejercicios con una marcada orientación postural, quizá no requiere una excesiva movilización muscular y energética para llegar a producir cambios sustanciales en la composición corporal de mujeres ya físicamente activas como el caso de las jugadoras de rugby donde hay una fuerte predisposición a presentar grandes valores musculares (Rodríguez, F.J., Crovetto, Berral de la Rosa, Almagiá, Iturriaga, \& Rodríguez, F. 2012)

Como limitaciones del estudio encontramos: la falta de un grupo control y/o grupo experimental; el escaso tamaño de la 
muestra; la duración del programa, ya que si se aumentase su duración, posiblemente se modificasen algunas de las variables antropométricas y además hubiésemos tenido datos a medio plazo, sobre las ventajas e inconvenientes del programa.

Teniendo en cuenta los resultados obtenidos en este estudio, podemos concluir que un programa de ocho semanas de la $\mathrm{TH}$, realizado por jugadoras de rugby puede contemplarse como una herramienta de terapia física y preventiva para el SP de jóvenes deportistas. Hacen faltan más estudios para corroborar los efectos de un programa regular de la TH en deportistas y a largo plazo, ampliando los mismos también hacia otros grupos de población.

\section{Agradecimientos}

Los/as autores/as agradecemos la colaboración de las participantes en el estudio así como a la fisioterapeuta Bibiana Penas por su dedicación y apoyo en la realización de este estudio.

\section{Referencias}

Bernardes, B., Resende, A., Stupp, L., Oliveira, E., Castro, R., Bella, Z...\& Sartori, M.G. (2012). Efficacy of pelvic floor muscle training and hypopressive exercises for treating pelvic organ prolapse in women: randomized controlled trial. Sao Paulo Med J, 130(1), 5-9.

Bo, K. (2004). Urinary incontinence, pelvic floor dysfunction, exercise \& sport. Sports Med, 34(7), 451-464.

Brown, W.J. \& Miller, Y.D. (2001). Too wet to exercise? Leaking urine as a barrier to physical activity in women. J Sci Med Sport, 4(4), 373-8.

Cairns, M., Harrison, K. \& Wright, C. (2000). Pressure biofeedback: a useful tool in the quantification of abdominal muscular dysfunction? Physiotherapy, 86(3), 127-138.

Caufriez, M., Fernández, J.C., \& Brynhildsvoll, N. (2011). Preliminary study on the action of hypopressive gymnastics in the treatment of idiopathic scoliosis. Enferm Clin, 21(6), 354-358.

Da Roza, T., de Araujo, M., Viana, R., Viana, S., Jorge, R., Bø, K. \& Mascarenhas, T. (2012). Pelvic floor muscle training to improve urinary incontinence in young, nulliparous sport students: a pilot study. Int Urogynecol J, 23(8), 1069-1073.

Da Roza, T., Brandao, S., Oliveira, D., Mascarenhas, T., Parente, M., Duarte, J.A et al., (2015). Football practice and urinary incontinence: relation between morphology, function and biomechanics. J Biomechanics, 48(9) 1587-1592.

Dietz, H.P. \& Shek, C. (2008). Levator avulsión and grading of pelvic floor muscle strength. Int Urogynecol J Pelvic Floor Dysfunct, 19(5), 633-636.

Esparza, F. (1993). Manual de Cineantropometría. Monografías FEMEDE. Editor científico: Grupo Español de Cineantropometría (GREC). Pamplona: FEMEDE.

Eliasson, K., Edner, A. \& Mattsson, E. (2008). Urinary Incontinence in very young and mostly nulliparous women with a history of regular organised high-impact trampoline training: occurrence and risk factors. Int Urogynecol J, 19(5), 687-696.

Gabbet, T.J. (2007). Physiological and anthorpometric characteristics of elite women rugby league players. J St Cond Res, 21(3), 875-881.

Gabbet, T.J. (2008). Incidence of injury in junior rugby league players over four competitive seasons. J Sci Med Sport, 11(3), 323-328.

Gabbett, T., King, T. \& Jenkins, D. (2008). Applied physiology of rugby league. Sports Med., 38(2), 119-138.

Gabbett, T.J. (2003). Incidence of injury in semi-professional rugby league players. Br J Sports Med, 37(1), 36-44.

García-Soidán, J.L, López, J., Ogando, H., Fernández, A., Padrón, A, \& Prieto, J. (2014). Utility kinanthropometry and bioimpedance to guide body composition and habits of the players. Retos. Nuevas tendencias en Educación Física, Deporte y Recreación, 25, 117-119.

Goldstick, O. \& Constantini, N. (2014). Urinary incontinence in physically active women and female athletes. Br J Sports Med, 48(4), 296-298.

Heyward, V.H. \& Wagner DR. (2004). Applied body composition assessment. Human kinetics: Champaign (USA).

Hodges, P., Richardson, C. \& Jull, G. (1996). Evaluation of the relationship between laboratory and clinical tests of transversus abdominis function. Physiother Res Int, 1(1), 30-40.

Jones, B., Emmonds, S., Hind, K., Nicholson, G., Rutherford, Z. \& Till, K. (2015). Physcial qualities of international female rugby league players. $J$ Strength Cond Res, in press.

King, D.A., Hume, P.A., Milburn, P.D. \& Guttenbeil, D. (2010). Match and training injuries in rugby league. A review of published studies. Sports Med, 40(2), 163-178.

Kruger, J., Murphy, B. \& Heap, S. (2005). Alterations in levator ani in elite nulliparous athletes: a pilot study. Aust $N \mathrm{Z} \mathrm{J}$ Obstet Gynaecol, 45, 42-7.

Kruger, J., Dietz, H. \& Murphy, B. (2007). Pelvic floor function in elite nulliparous athletes. Ultrasound Obstet Gynecol, 30, 81-85.

Latorre, G., Seleme, M., Resende, A.P., Stupp, L. \& Berghmans, B. (2011). Hypopressive gymnastics: evidences for an alternative training for women with local proprioceptive deficit of the pelvic floor muscles. Fisioterapia Brasil, 12(6), 463-466.

Mills, J., Taunton, J. \& Mills, W. (2005). The effect of a 10week training regimen on lumbo-pelvic stability and athletic performance in female athletes: A randomized-controlled trial. Phys Ther Sport, 6(2), 60-66.

Nygaard, I. (1997). Does Prolonged High-impact Activity Contribute to Later Urinary Incontinence? A Retrospective Cohort Study of Female Olimpians. Obstet Gynecol, 5(90), 718-722.

Resende, A., Stüpp, L., Bernardes, B., Oliveira, E., Castro, R., Girão, C., \& Sartori, M.G. (2011). Can hypopressive exercises provide additional benefits to pelvic floor muscle training in women with pelvic organ prolapse? Neurourol Urodyn, 31(1), 121-125.

Rial, T. \& Pinsach, P. (2014). Técnicas Hipopresivas. Ediciones Cardeñoso: Vigo.

Rial, T., Chulvi-Medrano, I., Cortell-Tormo, J.M., Álvarez, M. (2015). ¿Puede un programa de ejercicio basado en técnicas hipopresivas mejorar el impacto de la incontinencia urinaria en la calidad de vida de la mujer? Suelo Pélvico, 11(2), 2732.

Rodríguez, F.J., Crovetto M., Berral de la Rosa, F.J., Almagiá, A.A., Iturriaga, M.F. \& Rodríguez, F. (2012). Comparación de la composición corporal y de la masa muscular por segmentos corporales en estudiantes de educación física y deportistas de distintas disciplinas. Int J Morphol, 30(1), 714.

Stüpp, L., Resende, A., Petricelli, C., Nakamura, M., Alexandre, S. \& Zanetti, M. (2011). Pelvic floor muscle and transversus abdominis activation in abdominal hypopressive technique through surface electromyography. Neurourol Urodyn, 30(8), 518-521.

Wilkerson, G.B., Giles. J.L. \& Seibel. D.K. (2012). Prediction of core and lower extremity strains and sprains in collegiate football players: a preliminary study. J Athl Training, 47(3), 264-272.

Zazulak, B., Cholewicki, J. \& Reeves, N.P. (2008). Neuromuscular control of trunk stability clinical implications for sports injury prevention. J Am Acad Orthop Surg, 16(9), 497-505. 